\begin{tabular}{|c|l|}
\hline Title & An ab initio molecular dynamics study on the dissociative recombination reaction of HD2O+ + e- \\
\hline Author(s) & Kay anuma, Megumi; Taketsugu, Tetsuya; Ishii, Keisaku \\
\hline Citation & $\begin{array}{l}\text { Theoretical Chemistry Accounts, 120(1-3), 191-198 } \\
\text { https://doi.org/10.1007/300214007-03146 }\end{array}$ \\
\hline Issue Date & 2008 05 \\
\hline Doc URL & http://hdl.handle.net/2115/34137 \\
\hline Rights & The original publication is available at www.springerlink.com. \\
\hline Type & article (author version) \\
\hline File Information & HD20+.pdf \\
\hline
\end{tabular}

Instructions for use 


\title{
An ab initio molecular dynamics study
}

\section{on the dissociative recombination reaction of $\mathrm{HD}_{2} \mathrm{O}^{+}+\mathrm{e}^{-}$}

\author{
Megumi Kayanuma ${ }^{a}$, Tetsuya Taketsugu ${ }^{\text {b }}$, and Keisaku Ishii ${ }^{\mathrm{c}}$ \\ ${ }^{a}$ Department of Chemistry, Ochanomizu University, Tokyo 112-8610, Japan \\ ${ }^{\mathrm{b}}$ Division of Chemistry, Graduate School of Science, Hokkaido University, Sapporo 060-0810, Japan \\ ${ }^{\mathrm{c}}$ Department of Chemical System Engineering, Graduate School of Engineering, University of Tokyo, \\ Tokyo 113-8656, Japan
}

Corresponding author: Tetsuya Taketsugu

Tel \& Fax: +81 117063535

E-mail address: take@sci.hokudai.ac.jp 


\begin{abstract}
An ab initio molecular dynamics simulations have been carried out for the dissociative recombination reaction of the deuterium-substituted hydronium cation, $\mathrm{HD}_{2} \mathrm{O}^{+}+\mathrm{e}^{-}$, at the state-averaged multiconfigurational self-consistent field (SA-MCSCF) level. In the present simulations, five electronic states of $\mathrm{HD}_{2} \mathrm{O}$ were included explicitly, and nonadiabatic transitions among adiabatic electronic states were taken into account by the Tully's fewest switches algorithm. It is shown that the dominant products, $\mathrm{OD}+\mathrm{D}+\mathrm{H}$, were generated in $63 \%$ of trajectories, while the products, $\mathrm{OH}+$ $2 \mathrm{D}$, were generated in only $11 \%$ of trajectories, indicating that the release of a light fragment $\mathrm{H}$ is favored over the release of a heavy fragment D. This result is in conformity with the observation that there is a larger amount of deuterium substituted species than the non-substituted species in the interstellar space.
\end{abstract}

Keywords: Surface hopping, molecular dynamics, interstellar molecule, dissociative recombination, nonadiabatic coupling 


\section{Introduction}

Recent developments in computational performance for the last decade make it possible to combine ab initio electronic structure calculations with dynamics. In 1995, an option of ab initio molecular dynamics code (referred to as DRC) has been implemented in the ab initio program package, GAMESS [1]. This method was applied to several polyatomic reactions, in which the trajectories were analyzed in terms of normal coordinates of the molecule [2-5], or in terms of reaction coordinate and curvature coordinate $[6,7]$. This option enables one to carry out classical trajectory simulations for any molecular system in the electronic ground state, based on the energy gradients evaluated by ab initio molecular orbital calculations. The advantage of this approach is no requirement of potential energy functions in advance, while the disadvantage may be a huge computational cost compared to the conventional trajectory calculations using potential functions. Owing to the growth of performance of computer resources, however, the number of applications of this approach increases year by year, and it is now feasible to do direct trajectory simulations in the electronic ground state. The next stage may be to develop an ab initio molecular dynamics method for electronic excited states. In order to perform a trajectory simulation in electronic excited states, the energy gradients on the excited potential energy surface need to be evaluated. In general, a multiconfigurational self-consistent field (MCSCF) wavefunction is required to describe electronic excited states. Also, if one considers the possibility of nonadiabatic transitions between two adiabatic states, the state-averaged MCSCF (SA-MCSCF) wavefunctions, or higher ab initio multireference theory, should 
be employed. Very recently we have developed a program code for ab initio molecular dynamics simulations in the electronic excited states, including Tully's surface hopping algorithm [8]. This code was successfully applied to the dissociative recombination (DR) reactions of $\mathrm{HCNH}^{+}+\mathrm{e}^{-}$[9] and of $\mathrm{H}_{3} \mathrm{O}^{+}+\mathrm{e}^{-}[10]$ by the SA-MCSCF method.

The DR reaction is a combination of an electron and a positive molecular ion, which is followed by the dissociation into neutral molecules. Through the DR reactions, polyatomic ions dissociate into different combinations of neutral atoms/molecules, and the study of the branching ratios of the DR reaction will help to understand the chemical evolution in interstellar clouds [11-13]. The possible mechanism to generate the water molecule in interstellar clouds is the DR reaction of $\mathrm{H}_{3} \mathrm{O}^{+}$with electrons [14-17]. Jensen et al. investigated the branching ratios of the dissociative products from this reaction by using the heavy-ion storage ring ASTRID, and reported the following ratio $[18,19]$ :

$$
\mathrm{H}_{3} \mathrm{O}^{+}+\mathrm{e}^{-} \rightarrow\left\{\begin{array}{l}
\mathrm{OH}+2 \mathrm{H} \\
\mathrm{H}_{2} \mathrm{O}+\mathrm{H} \\
\mathrm{OH}+\mathrm{H}_{2} \\
\mathrm{O}+\mathrm{H}_{2}+\mathrm{H}
\end{array}\right.
$$

This branching ratio was deduced from the collision with zero relative energy of $\mathrm{H}_{3} \mathrm{O}^{+}$and a colliding electron. In ab initio molecular dynamics simulations for this DR reaction [10], the experimental branching ratios are reproduced qualitatively, although the rates of $\mathrm{H}_{2} \mathrm{O}+\mathrm{H}$ and $\mathrm{OH}+\mathrm{H}_{2}$ are relatively small compared to the experimental ones.

In interstellar clouds, the relative ratio of abundances of singly-deuterated species to that of non-deuterated species is observed to be on the order of 0.01-0.1 for several molecular species [20], 
which is much larger than the total cosmic abundance ratio of deuterium to hydrogen, $1 \times 10^{-5}[21]$. This observation indicates that the chemistry of interstellar clouds favors the formation of deuterated molecular species. Therefore, it is very interesting to examine the isotope effects on the branching ratios of dissociative products from the DR reactions. Jensen et al. reported the branching ratio from the DR reaction of deuterated species, $\mathrm{HD}_{2} \mathrm{O}^{+}$, as follows [19]:

$$
\mathrm{HD}_{2} \mathrm{O}^{+}+\mathrm{e}^{-} \rightarrow \quad \begin{cases}\mathrm{OD}+\mathrm{D}+\mathrm{H} & (0.42) \\ \mathrm{D}_{2} \mathrm{O}+\mathrm{H} & (0.15) \\ \mathrm{OH}+2 \mathrm{D} & (0.13) \\ \mathrm{HDO}+\mathrm{D} & (0.13) \\ \mathrm{OD}+\mathrm{HD} & (0.13) \\ \mathrm{OH}+\mathrm{D}_{2} & (0.03) \\ \mathrm{O}+\mathrm{HD}+\mathrm{D} & (0.01) \\ \mathrm{O}+\mathrm{D}_{2}+\mathrm{H} & (0.00)\end{cases}
$$

As is shown here, there are much more fragmentation channels than the non-substituted reaction in Eq.

(1), and the branching ratio of $\mathrm{OD}+\mathrm{D}+\mathrm{H}$ and $\mathrm{OH}+2 \mathrm{D}$ shows that the release of a lighter fragment $(\mathrm{H})$ is favored over the release of a heavier fragment (D). Based on a simple classical model, it was argued that if two electronically equivalent dissociation pathways that lead to different dissociation channels exist, pure kinetics tends to favor release of the lighter fragment. In addition, when dissociating along the pathway leading to release of lighter fragment, the neutral system has less time to decay by autoionization and hence a higher probability for completing the DR process [19].

In the present study, we apply the ab initio molecular dynamics method to the DR reaction of $\mathrm{HD}_{2} \mathrm{O}^{+}+\mathrm{e}^{-}$, to investigate the isotope effect in the DR reaction, and also to examine the reaction mechanism and the tendency in the branching ratio. 


\section{Energy diagram for DR reaction: $\mathrm{H}_{3} \mathrm{O}^{+}+\mathrm{e}^{-}$}

First we need to investigate an energy diagram for the $\mathrm{DR}$ reaction of $\mathrm{HD}_{2} \mathrm{O}^{+}+\mathrm{e}^{-}$to grasp the possible reaction pathways through electronic excited states, and also to check the accuracy of the ab initio methods employed in dynamics simulations. As is described in the introduction, we already performed similar calculations for $\mathrm{H}_{3} \mathrm{O}^{+}+\mathrm{e}^{-}$[10], and verified the accuracy of the method. Here we give a brief description as to ab initio electronic structure methods, and show the energy diagram for the DR reaction of $\mathrm{H}_{3} \mathrm{O}^{+}+\mathrm{e}^{-}$. The adiabatic potential energy surfaces are of course independent of atomic masses, and the energy diagram for $\mathrm{HD}_{2} \mathrm{O}^{+}+\mathrm{e}^{-}$is the same as that for $\mathrm{H}_{3} \mathrm{O}^{+}+\mathrm{e}^{-}$.

The energy diagram has been derived by the SA-MCSCF and following multireference Rayleigh-Schrödinger perturbation theory with contraction (RS2C) [22] methods for the ground and excited states of $\mathrm{H}_{3} \mathrm{O}$ and the dissociative products, $\mathrm{OH}, \mathrm{H}, \mathrm{H}_{2} \mathrm{O}, \mathrm{H}_{2}$, and $\mathrm{O}$, using the MOLPRO program package [23]. Geometry optimization was carried out for $\mathrm{H}_{3} \mathrm{O}^{+}$in the ground state at the MCSCF/6-311G(d,p) level with the full valence active space. For the thus optimized $\mathrm{H}_{3} \mathrm{O}^{+}$geometry $\left(C_{3 v}\right)$, the energetics were calculated for the neutral $\mathrm{H}_{3} \mathrm{O}$ in the ground state $\left(1^{2} \mathrm{~A}_{1}\right)$, the first excited degenerate state $\left({ }^{2} \mathrm{E}\right)$, and two Rydberg states (referred to as $\left.2{ }^{2} \mathrm{~A}_{1}, 3{ }^{2} \mathrm{~A}_{1}\right)$ with the 6-311G(d,p) basis sets augmented with Dunning-Hay's Rydberg functions of $O$ atom (s type function with $\alpha_{\mathrm{s}}=0.032$ and p type function with $\left.\alpha_{p}=0.028\right)$ [24] which is referred to as $6-311 G(d, p)+\operatorname{Ryd}(s, p)$. In these SA-MCSCF calculations, the full valence orbitals plus two Rydberg orbitals of $\mathrm{O}$ atom with $a_{1}$ symmetry ( $\mathrm{s}$ and $\mathrm{p}_{\mathrm{z}}$ orbitals where z-axis is taken as the $C_{3}$ axis of $\mathrm{H}_{3} \mathrm{O}^{+}$) were included in the active 
space, and five electronic states described above $\left(1^{2} \mathrm{~A}_{1}, 1{ }^{2} \mathrm{E}, 2{ }^{2} \mathrm{~A}_{1}\right.$, and $\left.3{ }^{2} \mathrm{~A}_{1}\right)$ were equally averaged. For $\mathrm{H}_{2} \mathrm{O}, \mathrm{OH}$, and $\mathrm{H}_{2}$, we optimized geometrical structures by the MCSCF/6-311G(d,p) method with the full valence active space, and calculated the relative energies. The energy for $\mathrm{O}\left({ }^{3} \mathrm{P}\right)$ atom was also calculated by (three-states-averaged) SA-MCSCF and RS2C methods with 6-311G(d,p) basis sets, while the energy for $\mathrm{H}$ atom was set to an accurate value, -0.5 hartree. The relative energy of $\mathrm{H}_{3} \mathrm{O}^{+}$ $\left(\mathrm{X}^{1} \mathrm{~A}_{1}\right)$ and $\mathrm{H}_{3} \mathrm{O}\left(1^{2} \mathrm{~A}_{1}\right)$ was evaluated by the state specific MCSCF and RS2C methods with the full valence active space and 6-311G(d,p) basis sets.

In the ground state of $\mathrm{H}_{3} \mathrm{O}^{+}$, there are three unoccupied valence orbitals, $4 a_{1}$ and $2 e$, which are all anti-bonding orbitals between $\mathrm{O}$ and $\mathrm{H}$ atoms. In the valence electronic states of $\mathrm{H}_{3} \mathrm{O}$, an additional electron comes into one of these unoccupied orbitals of $\mathrm{H}_{3} \mathrm{O}^{+}: 4 a_{1}$ is singly occupied in the $1{ }^{2} \mathrm{~A}_{1}$ ground state, while $2 e$ is singly occupied in the $1{ }^{2} \mathrm{E}$ first-excited state. In SA-MCSCF and RS2C calculations, $\mathrm{p}_{\mathrm{z}}$-type Rydberg orbital is singly occupied in the $2{ }^{2} \mathrm{~A}_{1}$ Rydberg state, while s-type Rydberg orbital is singly occupied in the $3{ }^{2} \mathrm{~A}_{1}$ Rydberg state. Figure 1 shows a summary of relative energies of (a) the related electronic states of $\mathrm{H}_{3} \mathrm{O}\left(1{ }^{2} \mathrm{~A}_{1}, 1{ }^{2} \mathrm{E}, 2{ }^{2} \mathrm{~A}_{1}, 3{ }^{2} \mathrm{~A}_{1}\right)$ and $\mathrm{H}_{3} \mathrm{O}^{+}\left(\mathrm{X}^{1} \mathrm{~A}_{1}\right)$, and (b) the dissociative products, $\mathrm{OH}+2 \mathrm{H}, \mathrm{H}_{2} \mathrm{O}+\mathrm{H}, \mathrm{OH}+\mathrm{H}_{2}$, and $\mathrm{O}+\mathrm{H}_{2}+\mathrm{H}$, at the SA-MCSCF and RS2C levels. The energy of $\mathrm{H}_{3} \mathrm{O}\left({ }^{2} \mathrm{~A}_{1}\right)$ at the equilibrium structure of $\mathrm{H}_{3} \mathrm{O}^{+}\left(\mathrm{X}^{1} \mathrm{~A}_{1}\right)$ is taken as zero at both the SA-MCSCF and RS2C levels in Figs. 1a and 1b. The MCSCF method gives a poor estimation of the relative energy of $\mathrm{H}_{3} \mathrm{O}^{+}$and $\mathrm{H}_{3} \mathrm{O}$, i.e., an ionization potential of $\mathrm{H}_{3} \mathrm{O}$, which is estimated as $71 \mathrm{kcal} / \mathrm{mol}$. The experimental ionization potential is reported as $4.3 \mathrm{eV}(=99.2$ 
$\mathrm{kcal} / \mathrm{mol}$ ) for $\mathrm{H}_{3} \mathrm{O}$ [25]. By including dynamical correlation effects in $\mathrm{RS} 2 \mathrm{C}$ calculations, the MCSCF energies of the electronic states of $\mathrm{H}_{3} \mathrm{O}$ are more lowered than the MCSCF energy of $\mathrm{H}_{3} \mathrm{O}^{+}$, resulting in more accurate energetics: ionization potential of $\mathrm{H}_{3} \mathrm{O}$ is estimated as $108 \mathrm{kcal} / \mathrm{mol}$ at the RS2C level.

As shown in Fig. 1, the MCSCF energies are in qualitatively agreement with the RS2C energies except for $\mathrm{H}_{3} \mathrm{O}^{+}$, although the RS2C energies for the dissociative products are higher by $12 \sim 26$ $\mathrm{kcal} / \mathrm{mol}$ than the MCSCF energy levels. Taking into account a qualitative agreement between SA-CASSCF and RS2C relative energies, we employ the SA-MCSCF method with the 6-311G(d,p)+Ryd(s,p) basis sets in the succeeding ab initio molecular dynamics simulations. 


\section{Ab initio molecular dynamics simulations}

$\mathrm{Ab}$ initio molecular dynamics simulations have been carried out for the $\mathrm{DR}$ reaction of $\mathrm{HD}_{2} \mathrm{O}^{+}+$ $\mathrm{e}^{-}$. In the present simulation, the trajectory proceeds on a single adiabatic potential energy surface (PES), and the surface hopping is invoked around the nonadiabatic region where two adiabatic PES's are close to each other. The atomic positions and velocities are developed by the Newton equation of motion, while the electronic degree of freedom is developed by the time-dependent Schrödinger equation. The forces acting on the respective atoms are evaluated as the negatives of the energy gradients of the PES that are determined by ab initio molecular orbital calculations [2-5]. As the surface-hopping scheme, we use the Tully's "fewest switches" algorithm [8].

In the present study we consider five electronic states of $\mathrm{HD}_{2} \mathrm{O}$, i.e., $1^{2} \mathrm{~A}, 2{ }^{2} \mathrm{~A}, 3^{2} \mathrm{~A}, 4{ }^{2} \mathrm{~A}$, and 5 ${ }^{2} \mathrm{~A}$, in $C_{1}$ point group where $4{ }^{2} \mathrm{~A}$ and $5{ }^{2} \mathrm{~A}$ correspond to the Rydberg states. The electronic wavefunction $\Psi$ is written as a function of time $t$,

$$
\Psi(t)=c_{1}(t) \Psi_{1}+c_{2}(t) \Psi_{2}+c_{3}(t) \Psi_{3}+c_{4}(t) \Psi_{4}+c_{5}(t) \Psi_{5},
$$

where $c_{k}(t)$ and $\Psi_{k}$ denote the electronic amplitude and eigenfunction, respectively, for the $k$ th ${ }^{2} \mathrm{~A}$ electronic state. Along the trajectory, the electronic amplitudes are also developed according to the time-dependent Schrödinger equation as 


$$
\left.\left[\begin{array}{l}
\dot{c}_{1}(t) \\
\dot{c}_{2}(t) \\
\dot{c}_{3}(t) \\
\dot{c}_{4}(t) \\
\dot{c}_{5}(t)
\end{array}\right]=\left(\begin{array}{ccccc}
\frac{V_{1}}{i \hbar} & -\mathbf{v} \cdot \mathbf{d}_{12} & -\mathbf{v} \cdot \mathbf{d}_{13} & -\mathbf{v} \cdot \mathbf{d}_{14} & -\mathbf{v} \cdot \mathbf{d}_{15} \\
\mathbf{v} \cdot \mathbf{d}_{12} & \frac{V_{2}}{i \hbar} & -\mathbf{v} \cdot \mathbf{d}_{23} & -\mathbf{v} \cdot \mathbf{d}_{24} & -\mathbf{v} \cdot \mathbf{d}_{25} \\
\mathbf{v} \cdot \mathbf{d}_{13} & \mathbf{v} \cdot \mathbf{d}_{23} & \frac{V_{3}}{i \hbar} & -\mathbf{v} \cdot \mathbf{d}_{34} & -\mathbf{v} \cdot \mathbf{d}_{35} \\
\mathbf{v} \cdot \mathbf{d}_{14} & \mathbf{v} \cdot \mathbf{d}_{24} & \mathbf{v} \cdot \mathbf{d}_{34} & \frac{V_{4}}{i \hbar} & -\mathbf{v} \cdot \mathbf{d}_{45} \\
\mathbf{v} \cdot \mathbf{d}_{15} & \mathbf{v} \cdot \mathbf{d}_{25} & \mathbf{v} \cdot \mathbf{d}_{35} & \mathbf{v} \cdot \mathbf{d}_{45} & \frac{V_{5}}{i \hbar}
\end{array}\right] \begin{array}{c}
c_{1}(t) \\
c_{2}(t) \\
c_{3}(t) \\
c_{4}(t) \\
c_{5}(t)
\end{array}\right],
$$

where $\mathbf{v}$ denotes atomic velocities, $\mathbf{d}_{k j}\left(=<\Psi_{k}|\nabla| \Psi_{j}>\right)$ denotes nonadiabatic coupling terms between the $k$ th and $j$ th electronic states, $V_{k}$ is the adiabatic potential energy of the $k$ th electronic state, and $\hbar$ is the Planck's constant divided by $2 \pi$. Nonadiabatic coupling terms are calculated by the SA-MCSCF method at each step. To reduce the computational cost, only the nonadiabatic coupling terms between neighboring states, $\mathbf{d}_{i, i+1}$, are evaluated, and others are set to zero. The probability of surface hopping from the $k$ th to the $j$ th electronic states within the time step of $\Delta t$ are evaluated from the electronic amplitudes and nonadiabatic coupling terms as [8],

$$
P_{k j}(t)=-2 \operatorname{Re}\left\{\left(\mathbf{v} \cdot \mathbf{d}_{k j}\right) c_{k} c_{j}^{*}\right\} \Delta t /\left|c_{k}\right|^{2}
$$

The surface hopping is invoked when $P_{k j}(t)$ is greater than a uniform random number generated between 0 and 1 . To conserve the total energy, the energy difference in the adiabatic potential energy before and after the surface hopping is converted to the kinetic energy. This conversion is performed by scaling the component of atomic velocities in the direction of the nonadiabatic coupling vector in mass-weighted coordinates.

In the direct trajectory simulations, the SA-MCSCF calculations were carried out, step by step, in which five ${ }^{2} A$ states are averaged with equal weights, and full-valence plus two Rydberg orbitals are 
included in the active space. The energy gradients and nonadiabatic coupling terms were calculated analytically by solving the coupled-perturbed multiconfigurational SCF equations for the relevant excited states. Such a direct trajectory approach requires extensive computational costs compared to the conventional trajectory simulations using potential energy functions. In this study, each trajectory has been run over a time length of up to $100 \mathrm{fs}$ until bond dissociation occurs, with a fixed time step of $0.1 \mathrm{fs}$. The criteria for bond dissociations were determined arbitrarily, by checking energy variations along the respective bonds, as follows:

$$
\begin{aligned}
& \mathrm{OH}+2 \mathrm{H} \quad \text { if two } r(\mathrm{OH})>3 \AA \text {, the other one } r(\mathrm{OH})<2 \AA \text {, and all } r(\mathrm{HH})>2 \AA, \\
& \mathrm{H}_{2} \mathrm{O}+\mathrm{H} \quad \text { if one } r(\mathrm{OH})>3 \AA \text { and the other two } r(\mathrm{OH})<3 \AA, \\
& \mathrm{OH}+\mathrm{H}_{2} \quad \text { if two } r(\mathrm{OH})>3 \AA \text {, the other one } r(\mathrm{OH})<2 \AA \text {, and one } r(\mathrm{HH})<2 \AA, \\
& \mathrm{O}+\mathrm{H}_{2}+\mathrm{H} \quad \text { if all } r(\mathrm{OH})>3 \AA \text { and one } r(\mathrm{HH})<2 \AA .
\end{aligned}
$$

The initial conditions for atomic positions and velocities are determined by the quasiclassical trajectory sampling [25] where zero-point vibrational energies are assigned, with random phases, to the respective normal modes of vibration of $\mathrm{HD}_{2} \mathrm{O}^{+}$in the electronic ground state. The sum of zero-point vibrational energy was evaluated as $18.0 \mathrm{kcal} / \mathrm{mol}$ at the MCSCF level. Then, the molecule is placed on the adiabatic $5{ }^{2} \mathrm{~A}$ PES of neutral $\mathrm{HD}_{2} \mathrm{O}$, and the electronic amplitudes at $t=0$ were set as,

$$
c_{1 \mathrm{~A}}=c_{2 \mathrm{~A}}=c_{3 \mathrm{~A}}=c_{4 \mathrm{~A}}=0, c_{5 \mathrm{~A}}=1 .
$$

It is noted that the $\mathrm{HD}_{2} \mathrm{O}^{+}$ground state and the $5^{2} \mathrm{~A}$ Rydberg state are accidentally degenerate with 
each other at the equilibrium point of $\mathrm{HD}_{2} \mathrm{O}^{+}$at the $\mathrm{RS} 2 \mathrm{C}$ level, as shown in Fig. 1. Then, the initial conditions described above correspond to the situation that $\mathrm{HD}_{2} \mathrm{O}^{+}$makes transitions to the $5{ }^{2} \mathrm{~A}$ Rydberg state of $\mathrm{HD}_{2} \mathrm{O}$ without any increase or decrease of the energy on capturing an electron. These conditions correspond to the experimental ones [19]. First we generated 99 different atomic coordinates and velocities by the quasiclassical trajectory sampling, and found that the SA-MCSCF energy of the $5{ }^{2} \mathrm{~A}$ state is too high in five initial molecular configurations. Thus, we carried out molecular dynamics simulations for the remaining 94 initial conditions.

In the ab initio molecular dynamics simulations with the SA-MCSCF method, we sometimes encounter a convergence problem in MCSCF calculations. This is partly because the SA-MCSCF wavefunction depends on the number of averaged states. The character of low-lying electronic states can change in different regions of the configurational space, indicating the difficulty to determine the constant number of averaged states. In the present simulations, the sixth electronic state in the external space often comes close to the fifth electronic state in the averaged states, invoking the convergence problem in SA-MCSCF calculations. When this situation occurs, we attempt to converge the SA-MCSCF calculation by applying several different orbital sets as initial guess; if these attempts failed, we increased the number of the averaged states to six temporarily, and continued the simulation until $5{ }^{2} \mathrm{~A}$ and $6{ }^{2} \mathrm{~A}$ states were separated sufficiently with each other; then, setting the number of averaged states to five again, we continued the simulation. If we encounter this situation while the molecule stays on the other states, $1{ }^{2} \mathrm{~A} \sim 4{ }^{2} \mathrm{~A}$, we decreased the number of averaged states 
one by one to continue the trajectory simulations. Even if such a convergence problem does not occur, we reduced the number of averaged states from 5 to 3 and eliminated two outer Rydberg orbitals from the active space after the molecule transits to the $3{ }^{2} \mathrm{~A}$ state, to reduce the computational costs. At these points, the potential energy shows a slightly discontinuous change due to the change of the SA-MCSCF wavefunctions described above. The energy corresponding to the change in potential energy is added to (or subtracted from) the total energy which should be conserved along the trajectory. 


\section{Results and discussion}

As is described in the previous section, 94 trajectories have been run on the $5^{2} \mathrm{~A}$ electronic state of $\mathrm{H}_{3} \mathrm{O}$ neutral species with the possibility of nonadiabatic transitions to different electronic states, starting from the zero-point-vibrational energy region of the electronic ground state of $\mathrm{H}_{3} \mathrm{O}^{+}$. The surface hopping scheme was taken into account by the Tully's fewest switches algorithm [8]. Figure 2 shows examples of variations of (a) the adiabatic potential energies, (b) the norm of the respective electronic amplitudes, (c) the inner product of the atomic velocity and the nonadiabatic coupling vector, and (d) the $\mathrm{O}-\mathrm{H}$ (or O-D) interatomic distances in $\mathrm{HD}_{2} \mathrm{O}$ along the trajectory. This trajectory leads to the products, $\mathrm{OD}+\mathrm{H}+\mathrm{D}$, where $\mathrm{H}$ atom leaves from $\mathrm{O}$ atom by $3.0 \AA$ at $t=41.2 \mathrm{fs}$ and one $\mathrm{D}$ atom (denoted as $\mathrm{D}^{(1)}$ ) leaves at $t=49.4 \mathrm{fs}$ as shown in Fig. $2 \mathrm{~d}$. The surface hopping occurs at $t=$ 19.2 fs $\left(5^{2} \mathrm{~A} \rightarrow 4{ }^{2} \mathrm{~A}\right), t=19.3$ fs $\left(4^{2} \mathrm{~A} \rightarrow 3{ }^{2} \mathrm{~A}\right), t=29.0$ fs $\left(3{ }^{2} \mathrm{~A} \rightarrow 2^{2} \mathrm{~A}\right)$, and $t=48.2$ fs $\left(2^{2} \mathrm{~A} \rightarrow 1\right.$ ${ }^{2} \mathrm{~A}$ ), which are denoted by dashed lines in Fig. 2a. After hopping to $3{ }^{2} \mathrm{~A}$ state, the number of averaged states was reduced from 5 to 3 and the active space was reduced by eliminating two Rydberg orbitals as described above, so the adiabatic energies of $1^{2} \mathrm{~A}$ and $2{ }^{2} \mathrm{~A}$ states are not continuous at this point. The discontinuous point in adiabatic potential energies was also observed at $t=31.3 \mathrm{fs}$ where the character of $3{ }^{2} \mathrm{~A}$ state changes. When the surface hopping occurs, two adiabatic states come close to each other in energy as shown in Fig. 2a, the electronic amplitude changes drastically as shown in Fig. 2b, and the inner product of the atomic velocity and nonadiabatic coupling vector becomes large as shown in Fig. 2c. Therefore, the surface hopping is invoked around the nonadiabatic region, although the Tully's fewest switches algorithm permits the molecule to make 
transitions between adiabatic states at any region in the configurational space. As shown in Fig. 2a, the $1{ }^{2} \mathrm{~A}, 2{ }^{2} \mathrm{~A}$, and $3{ }^{2} \mathrm{~A}$ states become degenerate in the final stage. This is a common feature in energy profiles for trajectories leading to $\mathrm{OD}+\mathrm{D}+\mathrm{H}$ or $\mathrm{OH}+2 \mathrm{D}$, which can be related to different spin couplings in the dissociation limit [10].

Figure 3 shows distributions of (a) the time when OH (or OD) bond cleaves and (b) the time when surface hopping occurs. In Fig. 3a, $\mathrm{H}(1)$ and $\mathrm{H}(2)$ denote the first and second $\mathrm{H}$ (or D) atoms leaving from the molecular system, respectively. The peak time for the first leaving $\mathrm{H}$ atom is about $25 \sim 30 \mathrm{fs}$, while the peak time for the second leaving $\mathrm{H}$ atom is about $30 \sim 35 \mathrm{fs}$. Each peak time is later than the corresponding leaving time of $\mathrm{H}(1)$ or $\mathrm{H}(2)$ in $\mathrm{H}_{3} \mathrm{O}^{+}+\mathrm{e}^{-}$by ca. 5 fs $[10]$. It is also interesting to note that the first leaving fragment is $\mathrm{H}$ atom in 29 trajectories while $\mathrm{D}$ atom leaves first in 25 trajectories. These numbers indicate that $\mathrm{H}$ atom is more favored to be released than $\mathrm{D}$ atom. As shown in Fig. 3b, the first transition from $5{ }^{2} \mathrm{~A}$ to $4{ }^{2} \mathrm{~A}$ was invoked at early stage $(\sim 10 \mathrm{fs})$, and the following transitions to the ground state have completed at around $t=45 \mathrm{fs}$ in most cases. The dissociation into $\mathrm{D}_{2} \mathrm{O}+\mathrm{H}, \mathrm{HDO}+\mathrm{D}$, or $\mathrm{O}+\mathrm{D}_{2}+\mathrm{H}$ occurs on the ground state, while the dissociation into $\mathrm{OD}+\mathrm{D}+\mathrm{H}$ or $\mathrm{OH}+2 \mathrm{D}$ occurs on one of the triply-degenerate states. In the latter case, the upward surface-hopping was observed in some trajectories, i.e., $2{ }^{2} \mathrm{~A} \rightarrow 3{ }^{2} \mathrm{~A}$ in five trajectories, and 1 ${ }^{2} \mathrm{~A} \rightarrow 2{ }^{2} \mathrm{~A}$ in nine trajectories.

To examine the motions of dissociated fragments, we carried out energy partition analyses in which the total energy is divided into translational, rotational, and vibrational energies of the 
respective fragments. Here the OD vibrational energy was estimated from the O-D vibrational amplitude, based on the SA-MCSCF potential energy curve of OD. The excess energy of the initial $\mathrm{H}_{3} \mathrm{O}$ system is evaluated as $172 \mathrm{kcal} / \mathrm{mol}$ relative to the dissociation limit of $\mathrm{H}_{2} \mathrm{O}+\mathrm{H}$, and as 66 $\mathrm{kcal} / \mathrm{mol}$ relative to the dissociation limit of $\mathrm{OH}+2 \mathrm{H}$ at the MCSCF level, which are distributed to the energies of the respective fragments in the dissociation limit. It is noted that these numbers are reduced to 155 and $42 \mathrm{kcal} / \mathrm{mol}$ for $\mathrm{H}_{2} \mathrm{O}+\mathrm{H}$ and $\mathrm{OH}+2 \mathrm{H}$, respectively, at the RS2C level.

In the present simulations, the dominant products are $\mathrm{OD}+\mathrm{D}+\mathrm{H}$ which were generated in 34 trajectories. This result agrees with tendency in the experimental branching ratio given in Eq. (2). In these trajectories, most of the energy was distributed to the OD vibrational energy and translational energy of $\mathrm{H}$ and $\mathrm{D}$ atoms. The translational energy is varied in a range of $0 \sim 50 \mathrm{kcal} / \mathrm{mol}$ (the peak is $10 \sim 15 \mathrm{kcal} / \mathrm{mol}$ ) for $\mathrm{H}$ atom and $0 \sim 65 \mathrm{kcal} / \mathrm{mol}$ (the peak is $45 \sim 50 \mathrm{kcal} / \mathrm{mol}$ ) for $\mathrm{D}$ atom, and thus, $\mathrm{D}$ atom have much more translational energy than $\mathrm{H}$ atom. In the previous simulation for $\mathrm{H}_{3} \mathrm{O}^{+}$, the peak is $20 \sim 25 \mathrm{kcal} / \mathrm{mol}$ for dissociated $\mathrm{H}$ atoms [10]. In OD molecules, the translational energy is $3 \sim 7 \mathrm{kcal} / \mathrm{mol}$, which is larger than that of $\mathrm{OH}$ produced in the simulation for $\mathrm{H}_{3} \mathrm{O}^{+}$(less than 4 $\mathrm{kcal} / \mathrm{mol}$ ). The rotational energy is less than $1 \mathrm{kcal} / \mathrm{mol}$ in both OD and $\mathrm{OH}$. The OD vibration energy varies in the range of $0 \sim 50 \mathrm{kcal} / \mathrm{mol}$ (the peak is $5 \sim 10 \mathrm{kcal} / \mathrm{mol}$ ), which is lower than that of $\mathrm{OH}[10] . \quad$ It is noted that the OD binding energy was calculated as ca. $80 \mathrm{kcal} / \mathrm{mol}$ at the SA-MCSCF level, so the succeeding dissociation will not occur.

There were 6 trajectories leading to $\mathrm{OH}+2 \mathrm{D}$ in which most of the energy was distributed to the 
$\mathrm{OH}$ vibrational energy and the translational energy of $\mathrm{D}$ atoms. The translational energies of $\mathrm{D}$ atoms vary in a range of $10 \sim 40$ and $0 \sim 30 \mathrm{kcal} / \mathrm{mol}$ for the first and second dissociating $\mathrm{D}$ atoms, respectively. In $\mathrm{OH}$ molecule, the translational and rotational energies are both less than $10 \mathrm{kcal} / \mathrm{mol}$. The $\mathrm{OH}$ vibrational energy varies in the range of $5 \sim 60 \mathrm{kcal} / \mathrm{mol}$ and so the dissociation to $\mathrm{O}+\mathrm{H}$ will not occur.

There were 10 trajectories that lead to the products, $\mathrm{HDO}+\mathrm{D}$, in which the translational energies are estimated in the range of $23 \sim 73 \mathrm{kcal} / \mathrm{mol}(21 \sim 66 \mathrm{kcal} / \mathrm{mol}$ for D and $2 \sim 7 \mathrm{kcal} / \mathrm{mol}$ for HDO). Those numbers are similar to those of $\mathrm{H}_{2} \mathrm{O}+\mathrm{H}$ in the simulation for $\mathrm{H}_{3} \mathrm{O}(9 \sim 81 \mathrm{kcal} / \mathrm{mol})$ [10]. The remaining energies, $99 \sim 149 \mathrm{kcal} / \mathrm{mol}$, should be attributed to the rotational and vibrational modes of HDO. The energy of $\mathrm{HDO}$ relative to that of $\mathrm{OD}+\mathrm{H}($ or $\mathrm{OH}+\mathrm{D})$ is evaluated as 106 $\mathrm{kcal} / \mathrm{mol}$ at the MCSCF level. Thus, the $\mathrm{H}_{2} \mathrm{O}$ molecule having more than $106 \mathrm{kcal} / \mathrm{mol}$ as the internal energy can possibly dissociate into $\mathrm{OD}+\mathrm{H}($ or $\mathrm{OH}+\mathrm{D})$ if the energy comes together to one $\mathrm{OH}$ (or OD) bond. Since HDO has a sufficient energy in seven of ten trajectories leading to $\mathrm{HDO}+\mathrm{H}$, it can further dissociate into $\mathrm{OD}+\mathrm{D}+\mathrm{H}$ or $\mathrm{OH}+2 \mathrm{D}$. There were 3 trajectories that lead to the product, $\mathrm{D}_{2} \mathrm{O}+\mathrm{H}$, in which the translational energies were estimated in the range of $21 \sim 30 \mathrm{kcal} / \mathrm{mol}(20 \sim 28$ $\mathrm{kcal} / \mathrm{mol}$ for $\mathrm{H}$ and $1 \mathrm{kcal} / \mathrm{mol}$ for $\mathrm{D}_{2} \mathrm{O}$ ). The remaining energies, $142 \sim 151 \mathrm{kcal} / \mathrm{mol}$, should be assigned to the rotational and vibrational modes of $\mathrm{D}_{2} \mathrm{O}$. The transitional energy is lower than that for $\mathrm{HDO}+\mathrm{D}$.

There is one trajectory leading to the products, $\mathrm{O}+\mathrm{D}_{2}+\mathrm{H}$, in which $\mathrm{H}$ atom attempt to leave 
after the transition to $2{ }^{2} \mathrm{~A}$ at $t=28.5 \mathrm{fs}$, and then two $\mathrm{D}$ atoms start escaping from $\mathrm{O}$ atom and bind together to become $\mathrm{D}_{2}$. The first $\mathrm{H}$ atom leaves from $\mathrm{O}$ atom by $3.0 \AA$ at $t=39.9 \mathrm{fs}$, while two $\mathrm{D}$ atoms leave by $3.0 \AA$ almost simultaneously at $t=51.5 \sim 55.2 \mathrm{fs}$. The vibrational energy for leaving $\mathrm{D}_{2}$ was calculated as ca. $27.5 \mathrm{kcal} / \mathrm{mol}$. It is noted that the branching ratio for $\mathrm{O}+\mathrm{D}_{2}+\mathrm{H}$ is zero in the experimental study as shown in Eq. (2).

Finally we show comparison of the branching ratios of dissociated fragments between theoretical ones obtained in the present simulation and experimental ones [19]. Among 94 trajectories, $\mathrm{H}_{3} \mathrm{O}$ keeps its all $\mathrm{OH}$ bonds throughout (no bond cleavage) in 40 trajectories, while some bond dissociations occur during $100 \mathrm{fs}$ in 54 trajectories. Among 54 trajectories, $\mathrm{HD}_{2} \mathrm{O}$ dissociates into $\mathrm{OD}+\mathrm{D}+\mathrm{H}$ in 34 trajectories, into $\mathrm{D}_{2} \mathrm{O}+\mathrm{H}$ in 3 trajectories, into $\mathrm{OH}+2 \mathrm{D}$ in 6 trajectory, into $\mathrm{HDO}$ $+\mathrm{D}$ in 10 trajectory, and into $\mathrm{O}+\mathrm{D}_{2}+\mathrm{H}$ in one trajectory, while $\mathrm{OD}+\mathrm{HD}, \mathrm{OH}+\mathrm{D}_{2}$, and $\mathrm{O}+\mathrm{HD}+\mathrm{D}$ are not produced. The branching ratios are as follows:

$$
\mathrm{HD}_{2} \mathrm{O}^{+}+\mathrm{e}^{-} \rightarrow\left\{\begin{array}{lcc} 
& \text { Experiment } & \text { Calculation } \\
\mathrm{OD}+\mathrm{D}+\mathrm{H} & (0.42) & (0.63) \\
\mathrm{D}_{2} \mathrm{O}+\mathrm{H} & (0.15) & (0.06) \\
\mathrm{OH}+2 \mathrm{D} & (0.13) & (0.11) \\
\mathrm{HDO}+\mathrm{D} & (0.13) & (0.18) \\
\mathrm{OD}+\mathrm{HD} & (0.13) & (0.00) \\
\mathrm{OH}+\mathrm{D}_{2} & (0.03) & (0.00) \\
\mathrm{O}+\mathrm{HD}+\mathrm{D} & (0.01) & (0.00) \\
\mathrm{O}+\mathrm{D}_{2}+\mathrm{H} & (0.00) & (0.02)
\end{array}\right.
$$

Of course the number of trajectories is very small in the present simulations, but the tendency of branching ratios can be discussed. As shown here, the qualitative agreement between theory and 
experiment has been obtained. The rate of $\mathrm{D}_{2} \mathrm{O}+\mathrm{H}$ is smaller while the rate for $\mathrm{HDO}+\mathrm{D}$ is slightly larger than the corresponding experimental rate. This may be caused by the difference in kinetic energy of dissociating fragments between $\mathrm{H}$ and $\mathrm{D}$ atoms. As discussed above, the dissociating $\mathrm{D}$ atoms tend to have more kinetic energy than the dissociating $\mathrm{H}$ atoms, and so the remaining energy in HDO is smaller than that in $\mathrm{D}_{2} \mathrm{O}$. The large excess energy in $\mathrm{D}_{2} \mathrm{O}$ might have caused a further dissociation to $\mathrm{OD}+\mathrm{D}$. It is noted that the relative energy of $\mathrm{H}_{2} \mathrm{O}+\mathrm{H}$ is estimated to be too low at the SA-CASSCF level as shown in Fig. 1. As another point, the difference in the relative ratio of OD $+\mathrm{D}+\mathrm{H}$ and $\mathrm{OH}+2 \mathrm{D}$ indicates that the isotope effect is essentially important in this DR reaction: $\mathrm{H}$ atom is favored to dissociate than $\mathrm{D}$ atom. This result supports the interstellar observation that the relative ratio of abundances of deuterated species to that of non-deuterated species is much larger than the ratio of $\mathrm{D}$ atom to $\mathrm{H}$ atom. 


\section{Concluding remarks}

$\mathrm{Ab}$ initio molecular dynamics simulations have been carried out for the dissociative recombination reaction, $\mathrm{HD}_{2} \mathrm{O}^{+}+\mathrm{e}^{-}$, at the SA-MCSCF level. The purposes are (1) an investigation of the tendency in the branching ratio of the dissociative products, (2) an examination of isotope effects in the DR reactions by comparing the results of similar simulations for $\mathrm{H}_{3} \mathrm{O}^{+}+\mathrm{e}^{-}$, and (3) getting insight to the dynamical processes of multi-state reactions with nonadiabatic transitions. The surface hopping mechanism was taken into account by the Tully's fewest switches algorithm, which permits nonadiabatic transitions between adiabatic states at any region in the configurational space. In the simulations two Rydberg states of $\mathrm{HD}_{2} \mathrm{O}$ have been included, which play a significant role in the indirect mechanism. It is verified that MCSCF and RS2C methods give the qualitatively similar energy diagram, although the MCSCF energies of the dissociative products were higher by $12 \sim 26$ $\mathrm{kcal} / \mathrm{mol}$ than the RS2C energies. We have run 94 trajectories with the initial conditions that zero-point vibrational energies are given to the respective normal vibrational modes of $\mathrm{HD}_{2} \mathrm{O}^{+}$with random phases, and the molecule is put on the second Rydberg state of $\mathrm{HD}_{2} \mathrm{O}$. In the present simulations, the surface hopping was observed in the relatively early stage less than $30 \mathrm{fs}$ in most cases. The rate of exothermic products is small compared to the experimental ones, but the tendency in the branching ratio is roughly reproduced. It is shown that the leaving $\mathrm{D}$ atoms tend to have more kinetic energy than the leaving $\mathrm{H}$ atoms, and that $\mathrm{H}$ atoms are favored to release than $\mathrm{D}$ atoms. Such isotope effects in the DR reactions should be a significant factor to determine the relative ratio of abundances of deuterated species to that of non-deuterated species in the interstellar space. 


\section{Acknowledgments}

This paper is dedicated to Professor Mark S. Gordon in celebration of his 65th birthday.

The present work was supported in part by a Grant-in-Aid for Scientific Research from Ministry of Education, Science and Culture, and was supported in part by Asian Office of Aerospace Research and

Development (AOARD). Present simulations were carried out by an allocation of computing resources of SGI2800 from the Institute of Statistical Mathematics in Tokyo. 


\section{References}

1 Schmidt MW, Baldridge KK, Boatz JA, Elbert ST, Gordon MS, Jensen JH, Koseki S, Matsunaga N,

Nguyen KA, Su SJ, Windus TL, Dupuis M, Montgomery JA (1993) J Comput Chem 14: $1347-1363$

2 Taketsugu T, Gordon MS (1995) J Phys Chem 99: 8462-8471

3 Taketsugu T, Gordon MS (1995) J Phys Chem 99: 14597-14604

4 Gordon MS, Chaban G, Taketsugu T (1996) J Phys Chem 100:11512-11525

5 Taketsugu T, Yanai T, Hirao K, Gordon MS (1998) J Mol Struc (THEOCHEM) 451: 163-177

6 Taketsugu T, Gordon MS (1995) J Chem Phys 103: 10042-10049

7 Taketsugu T, Gordon MS (1995) J Chem Phys 104: 2834-2840

8 Tully JC (1990) J Chem Phys 93:1061-1071

9 Taketsugu T, Tajima A, Ishii K, Hirano T (2004) Astrophys J 608: 323-329

10 Kayanuma M, Taketsugu T, Ishii K (2005) Chem Phys Lett 418:511-518

11 Smith D (1992) Chem Rev 92:1473-1485

12 Sternberg A, Dalgarno A (1995) Astrophys J Suppl 99:565-607

13 Herbst E, Lee H-H (1997) Astrophys J 485:689-696

14 Van Dishoeck EF (1990) in Molecular Astrophysics, ed. T. W. Hartquist, Cambridge University Press, Cambridge, UK, pp55

15 Miller TJ (1990) in Molecular Astrophysics, ed. T. W. Hartquist, Cambridge University Press, Cambridge, UK, pp115 
16 Bates DR (1990) in Molecular Astrophysics, ed. T. W. Hartquist, Cambridge University Press, Cambridge, UK, pp211

17 Neufeld DA, Lepp S, Melnick GJ (1995) Astrophys J Suppl 100:132-147

18 Vejby-Christensen L, Andersen LH, Heber O, Kella D, Pedersen HB, Schmit HT, Zajfman D (1997) Astrophys J 483:531-540

19 Jensen MJ, Bilodeau RC, Safvan CP, Seiersen K, Andersen LH, Pedersen HB, Heber O (2000) Astrophys J 543:764-774

20 Turner BE, Zuckerman B (1978) Astrophys J 225:L75-L79

21 Dalgarno A, Lepp D (1984) Astrophys J 287:L47-L50

22 Celani P, Werner H-J (2000) J Chem Phys 112:5546-5557

23 MOLPRO, a package of ab initio programs designed by Werner H-J, Knowles PJ, version 2002.1, Amos RD, Bernhardsson A, Berning A, Celani P, Cooper DL, Deegan MJO, Dobbyn AJ, Eckert F, Hampel C, Hetzer G, Knowles PJ, Korona T, Lindh R, Lloyd AW, McNicholas SJ, Manby FR, Meyer W, Mura ME, Nicklass A, Palmieri P, Pitzer R, Rauhut G, Schutz M, Schumann U, Stoll H, Stone AJ, Tarroni R, Thorsteinsson T, Werner H-J

24 Dunning Jr TH, Hay PJ (1977) in Methods of electronic structure theory, vol. 2, ed. H. F. Schaefer III, Plenum Press 25 Gellene GI, Porter RF (1984) J Chem Phys 81: 5570-5576

26 Peslherbe GH, Wang H, Hase WL (1999) in Advances in Chemical Physics, vol. 105, eds. Ferguson 
DM, Siepman JI, Truhlar DG, Wiley, New York, pp171-201 


\section{Figure caption}

Fig. 1. Relative energies of (a) the electronic states of $\mathrm{H}_{3} \mathrm{O}\left(1{ }^{2} \mathrm{~A}_{1}, 1{ }^{2} \mathrm{E}, 2{ }^{2} \mathrm{~A}_{1}, 3{ }^{2} \mathrm{~A}_{1}\right)$ and $\mathrm{H}_{3} \mathrm{O}^{+}\left(\mathrm{X}^{1} \mathrm{~A}_{1}\right)$, and (b) the dissociative products, $\mathrm{OH}+2 \mathrm{H}, \mathrm{H}_{2} \mathrm{O}+\mathrm{H}, \mathrm{OH}+\mathrm{H}_{2}$, and $\mathrm{O}+\mathrm{H}_{2}+$ $\mathrm{H}$, at the SA-MCSCF (or MCSCF) and RS2C levels.

Fig. 2. Variation of (a) the adiabatic potential energies, (b) the norm of the respective electronic amplitudes, (c) the inner product of the atomic velocity and nonadiabatic coupling vector, and (d) the O-H and O-D interatomic distances, along the trajectory.

Fig. 3. Distributions of (a) the lifetime of the trajectory until bond dissociations occur and (b) the time step when surface hopping in the descending way occurs. In part (a), H(1) and $\mathrm{H}(2)$ indicate the first and second hydrogen or deuterium atoms departing from oxygen atom. 

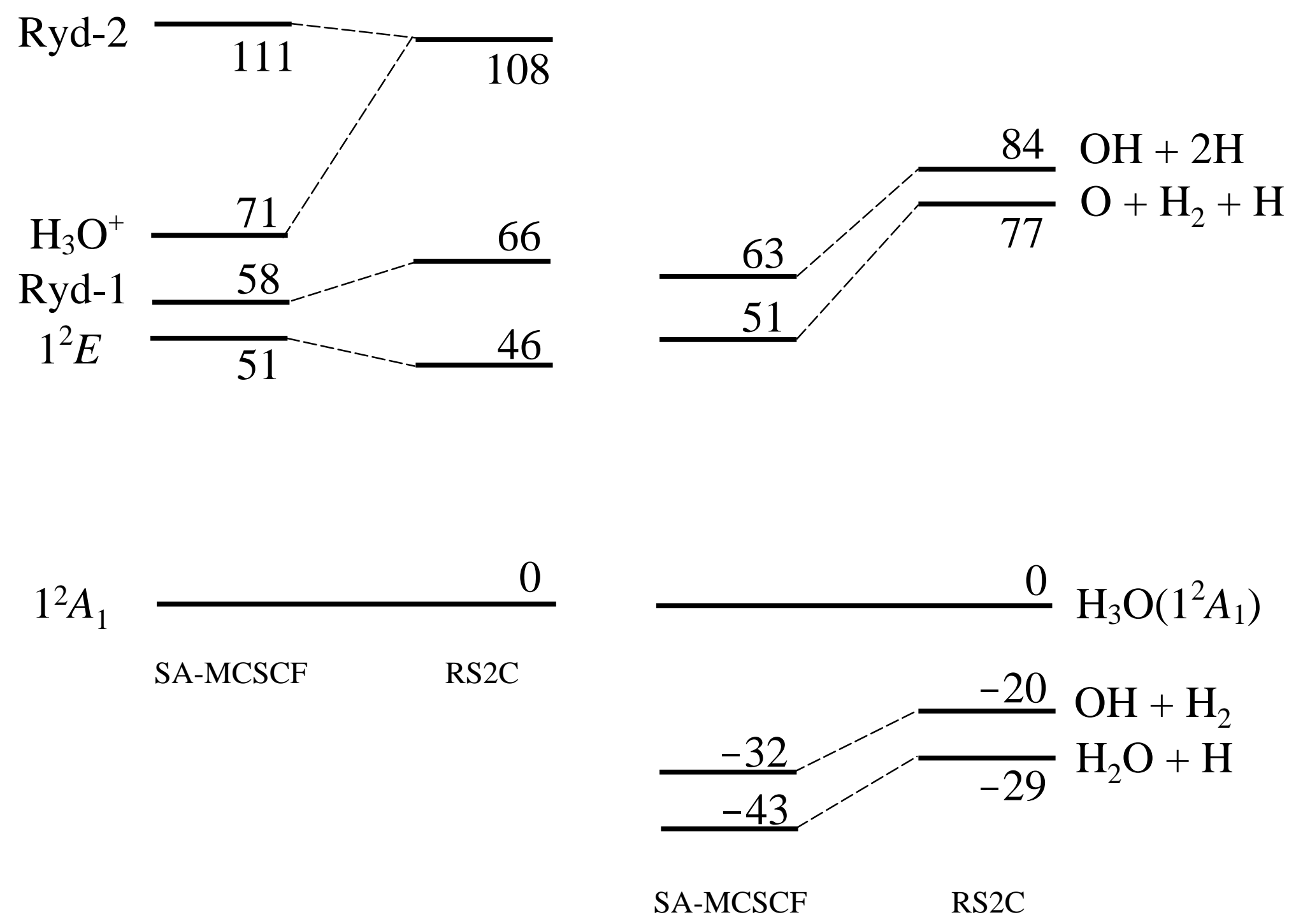

(a) Excited states of $\mathrm{H}_{3} \mathrm{O}$

(b) Dissociative products 
(a)

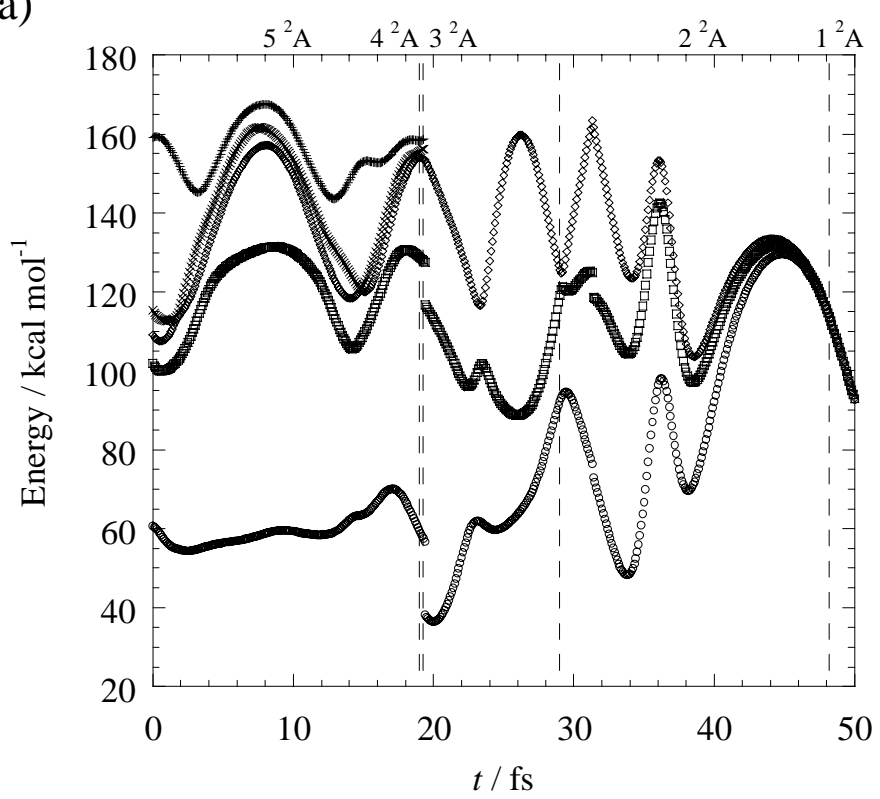

(c)

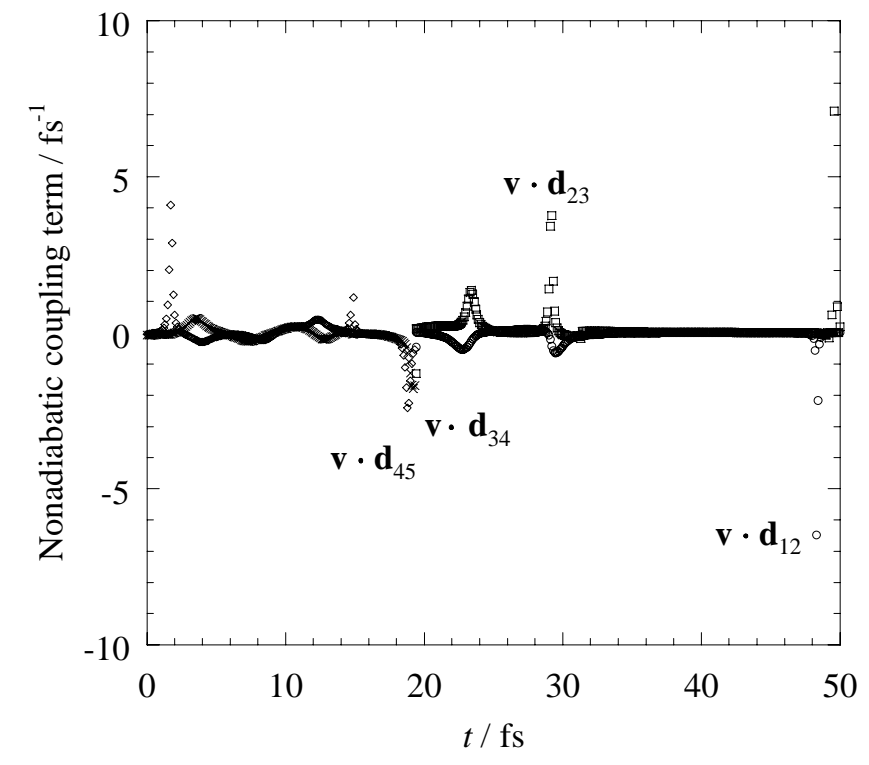

(b)

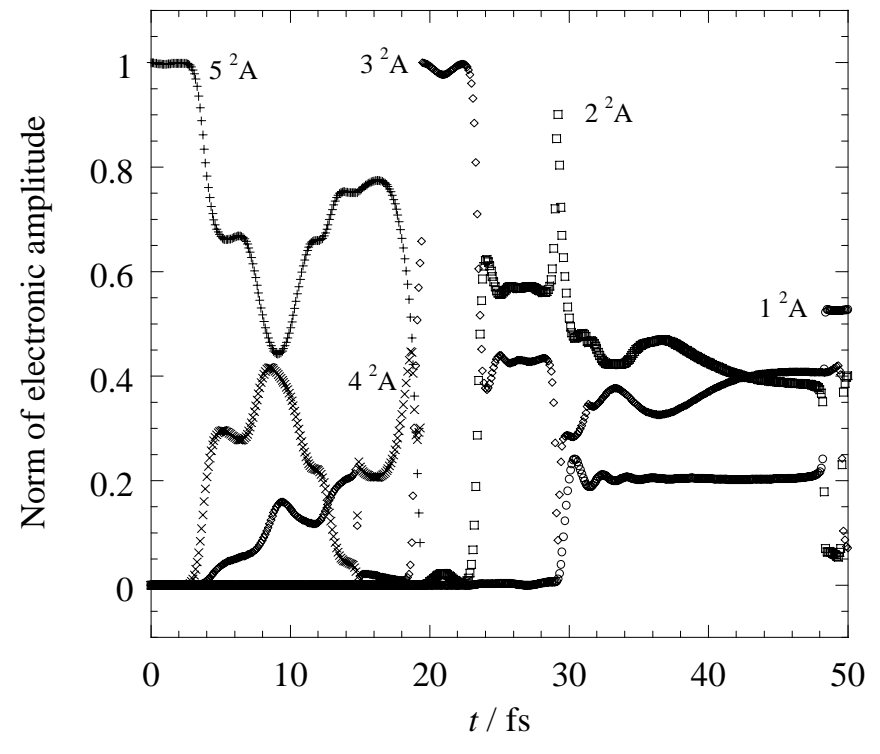

(d)

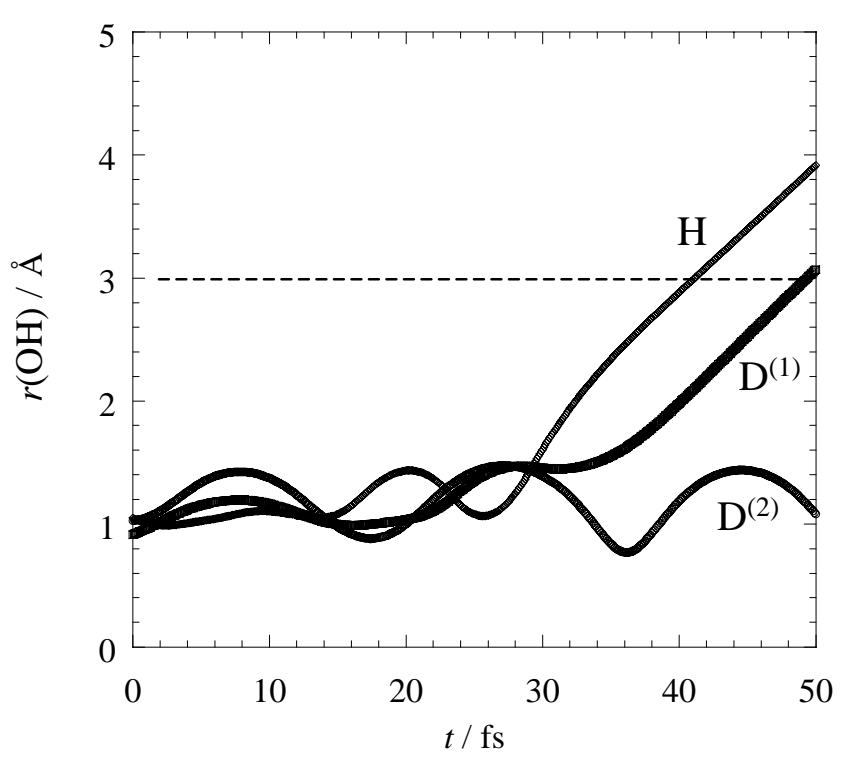

Fig.2. “An ab initio molecular dynamics ...” by Kayanuma et al. 
(a)

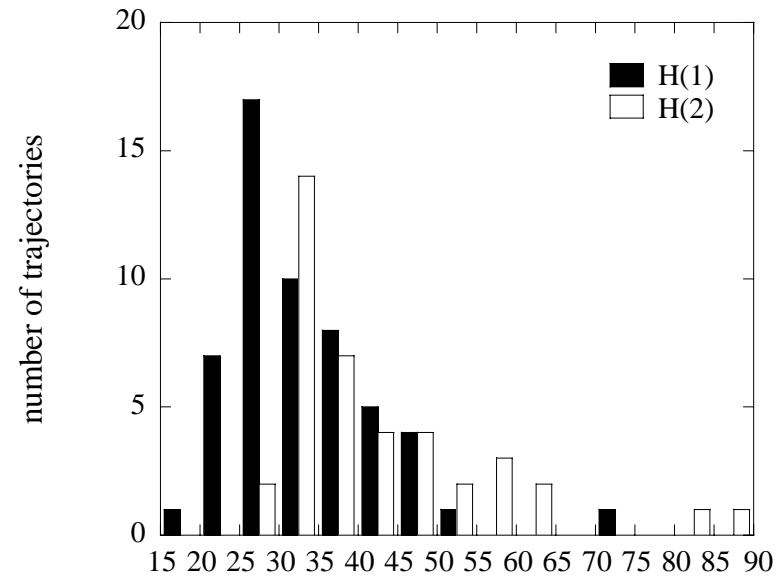

$\mathrm{t} / \mathrm{fs}$

(b)

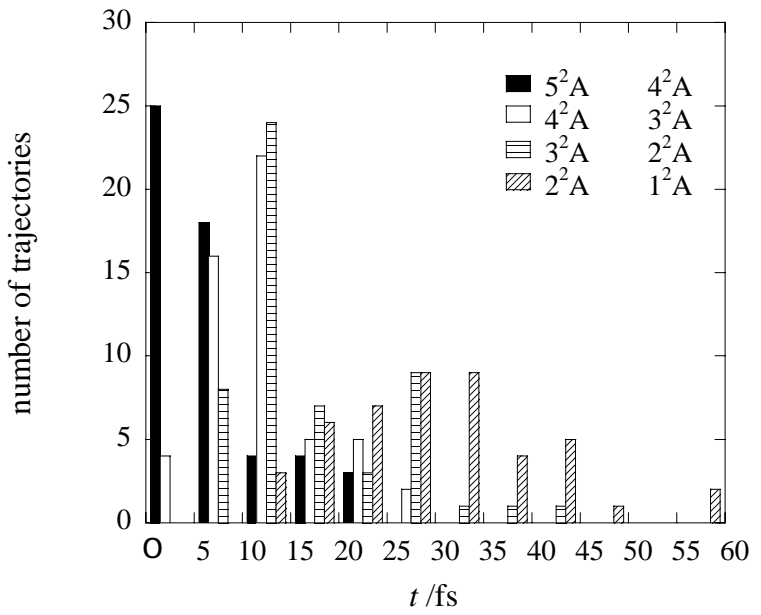

Fig.3. “An ab initio molecular dynamics ...” by Kayanuma et al. 